2010s-39

\title{
Servir les clients avec le sourire : un cadre motivationnel pour mieux prédire les stratégies de régulation émotionnelle
}

\author{
Marie-Claude Lépine, Michel Cossette
}

\begin{tabular}{c}
\hline Série Scientifique \\
Scientific Series
\end{tabular}

Montréal

Septembre 2010

(C) 2010 Marie-Claude Lépine, Michel Cossette. Tous droits réservés. All rights reserved. Reproduction partielle permise avec citation du document source, incluant la notice (C).

Short sections may be quoted without explicit permission, if full credit, including (C) notice, is given to the source.
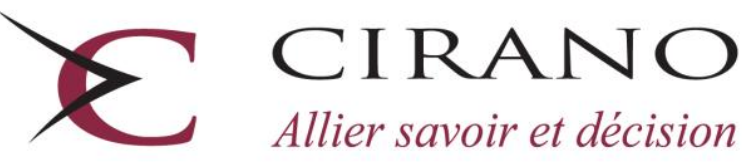

Allier savoir et décision

Centre interuniversitaire de recherche en analyse des organisations 


\section{CIRANO}

Le CIRANO est un organisme sans but lucratif constitué en vertu de la Loi des compagnies du Québec. Le financement de son infrastructure et de ses activités de recherche provient des cotisations de ses organisations-membres, d'une subvention d'infrastructure du Ministère du Développement économique et régional et de la Recherche, de même que des subventions et mandats obtenus par ses équipes de recherche.

CIRANO is a private non-profit organization incorporated under the Québec Companies Act. Its infrastructure and research activities are funded through fees paid by member organizations, an infrastructure grant from the Ministère du Développement économique et régional et de la Recherche, and grants and research mandates obtained by its research teams.

\section{Les partenaires du CIRANO}

Partenaire majeur

Ministère du Développement économique, de l'Innovation et de l'Exportation

\section{Partenaires corporatifs}

Banque de développement du Canada

Banque du Canada

Banque Laurentienne du Canada

Banque Nationale du Canada

Banque Royale du Canada

Banque Scotia

Bell Canada

BMO Groupe financier

Caisse de dépôt et placement du Québec

Fédération des caisses Desjardins du Québec

Gaz Métro

Hydro-Québec

Industrie Canada

Investissements PSP

Ministère des Finances du Québec

Power Corporation du Canada

Raymond Chabot Grant Thornton

Rio Tinto

State Street Global Advisors

Transat A.T.

Ville de Montréal

\section{Partenaires universitaires}

École Polytechnique de Montréal

HEC Montréal

McGill University

Université Concordia

Université de Montréal

Université de Sherbrooke

Université du Québec

Université du Québec à Montréal

Université Laval

Le CIRANO collabore avec de nombreux centres et chaires de recherche universitaires dont on peut consulter la liste sur son site web.

Les cahiers de la série scientifique (CS) visent à rendre accessibles des résultats de recherche effectuée au CIRANO afin de susciter échanges et commentaires. Ces cahiers sont écrits dans le style des publications scientifiques. Les idées et les opinions émises sont sous l'unique responsabilité des auteurs et ne représentent pas nécessairement les positions du CIRANO ou de ses partenaires.

This paper presents research carried out at CIRANO and aims at encouraging discussion and comment. The observations and viewpoints expressed are the sole responsibility of the authors. They do not necessarily represent positions of CIRANO or its partners. 


\title{
Servir les clients avec le sourire : un cadre motivationnel pour mieux prédire les stratégies de régulation émotionnelle
}

\author{
Marie-Claude Lépine ${ }^{*}$, Michel Cossette ${ }^{\dagger}$
}

\begin{abstract}
Résumé / Abstract
Le travail émotionnel, soit le fait pour les employés d'avoir à afficher ou à supprimer certaines émotions dans le cadre de leur travail, constitue une demande occupationnelle importante dans les emplois de service à la clientèle. Les stratégies de régulation émotionnelle ont des effets importants non seulement pour l'individu (attitudes au travail, intention de quitter son emploi et santé psychologique), mais aussi pour son organisation (roulement du personnel, performance de service, fidélisation de la clientèle, etc.). Bien que la motivation ait été proposée comme prédicteur du travail émotionnel, des enjeux de mesure de ce concept doivent être surmontés. Conséquemment, la présente étude propose et teste auprès d'employés travaillant avec des clients $(n=223)$ une échelle mesurant la motivation à réguler les émotions au travail. Des analyses factorielles confirmatoires soutiennent globalement la présence des types de motivation postulés par la théorie de l'autodétermination (Deci \& Ryan, 1985). De plus, la régulation émotionnelle de profondeur est liée à la motivation dite «contrôlante » et à la motivation «autonome», alors que la régulation de surface est seulement associée à la motivation « contrôlante».
\end{abstract}

Mots clés : Travail émotionnel, Motivation, Régulation émotionnelle

Emotional labour, a requirement imposed on employees to express or suppress their emotions in their job, is an important occupational demand in customer service jobs. Emotion regulation strategies has important impacts on individuals (job attitudes, intention to quit and psychological health), but also on their organization (personnel turnover, service performance, customers' loyalty, etc.). Although motivation was proposed as an emotional labour predictor, measurement issues of this concept must be resolved. Consequently, the present study proposes and tests among employees working in interaction with customers $(n=223)$ a scale measuring motivation to regulate emotions at work. Confirmatory Factor Analyses supported globally the presence of the different types of motivation postulated by the Self-determination Theory (Deci \& Ryan, 1985). Moreover, deep acting strategy was linked with the controlled motivation and to autonomous motivation, whereas surface acting was linked solely with controlled motivation.

Keywords: Emotional Labour, Motivation, Emotion regulation

\footnotetext{
*Étudiante à la M.Sc. (gestion des ressources humaines) à HEC Montréal, 3000, Chemin de la Côte SainteCatherine, Montréal (Québec), Canada H3T 2A7, courriel : mc_lepine@ hotmail.com

$\dagger$ Professeur adjoint au Service de l'enseignement de la GRH, HEC Montréal, 3000, Chemin de la Côte-SteCatherine, Montréal (Québec), Canada, H3T 2A7, tél. : 1-514-340-7038, fax : 1-514-340-6898, courriel : michel.cossette@hec.ca
} 


\section{Introduction}

L'expression des émotions joue un rôle essentiel dans le domaine du service à la clientèle (Hess, 2003). Au travail, les employés doivent réguler leurs émotions en fonction de ce qui est approprié pour la situation et de ce qui est attendu par l'employeur (Morris \& Feldman, 1997). Les entreprises déterminent des règles d'affichage émotionnel qui guident les employés dans leurs interactions avec la clientèle (Brotheridge \& Lee, 2002, 2003; Gosserand \& Diefendorff, 2005). Généralement, ils doivent exprimer des émotions positives et supprimer leurs émotions négatives (Brotheridge \& Grandey, 2002; Dienfendorff, Croyle \& Gosserand, 2005). Afin de se conformer aux règles d'affichage émotionnel, les employés utilisent diverses stratégies de régulation émotionnelle. Ainsi, le travail émotionnel correspond à l'action d'afficher l'émotion appropriée et de supprimer les émotions inappropriées, et ce, peu importe ce que l'individu ressent réellement (Ashforth \& Humphrey; 1993).

Les impacts du travail émotionnel sur les attitudes au travail et la santé psychologique des employés sont assez bien documentés (voir Bono \& Vey, 2005). En outre, parmi les antécédents connus du travail émotionnel figurent notamment les demandes émotionnelles de l'emploi, tels que la fréquence des émotions à afficher, la durée des interactions, la diversité des émotions à afficher (Brotheridge et Grandey, 2002; Morris \& Feldman, 1996a,b). Toutefois, le concept de motivation commence à être évalué dans le cadre de recherches récentes sur le travail émotionnel (Cossette, 2008, 2010; Cossette, Blais, \& Hess, 2006; Gosserand \& Diefendorff, 2005). Ce concept est porteur d'idées et de questions de recherche intéressantes, mais encore trop peu d'études se penchent la teneur et la nature de cette relation. Conséquemment, la présente recherche vise à opérationnaliser la motivation à accomplir le travail émotionnel et à vérifier le lien entre cette motivation et les stratégies de régulation émotionnelle.

\section{Une approche motivationnelle du travail émotionnel}

\subsection{Stratégies de régulation}

Le modèle du processus de régulation émotionnelle de Gross (1998a) catégorise les stratégies de régulation émotionnelle en deux classes distinctes (Figure 1). D'une part, certaines stratégies se centrent sur les réponses émotionnelles (les aspects observables des émotions et les manifestations physiologiques). D'autre part, d'autres stratégies focalisent sur les antécédents émotionnels (choix de la situation, interprétation ou évaluation de la situation, etc.).

La littérature portant sur le travail émotionnel se concentre principalement sur deux types de stratégies de régulation émotionnelle, soit la stratégie de régulation émotionnelle de surface (ci-dessous appelée régulation de surface) et la stratégie de régulation émotionnelle de profondeur (ci-dessous appelée régulation de profondeur). La stratégie de surface consiste à supprimer des émotions proscrites par l'organisation (typiquement des émotions négatives) et à simuler les émotions prescrites (faire semblant de sourire au client). Cette stratégie de régulation se concentre donc sur les réponses émotionnelles. L'objectif de ces stratégies est de modifier l'aspect observable de l'émotion (Hochschild, 1983; Gross, 1998a; Grandey, 2000; Grandey, 2003; Gross \& John, 2003, Chu \& Murrmann, 2006). Les stratégies de profondeur quant à elles sont centrées sur les antécédents ou les causes des émotions (Gross, 1998a, 1998b; Grandey, 2000; Gross \& John, 2003). Dans ce types de stratégies, la personne s'efforce à ressentir et à exprimer l'émotion souhaitée par l'entreprise, notamment en modifiant sa perception de la situation ou en réévaluant celle-ci (Hochschild, 1983; Grandey, 2000, 2003; Brotheridge \& Grandey, 2002; Brotheridge \& Lee, 2002; Diefendorff, Croyle \& Gosserand, 2005).

Pourquoi s'intéresser aux stratégies de régulation émotionnelle en milieu de travail? Parce que celles-ci entraînent des conséquences, notamment sur l'intention de quitter l'emploi (Côté \& Morgan, 
2002), l'épuisement professionnel (Kim, 2008) et la satisfaction au travail (Grandey, 2003). Globalement, la régulation de surface s'avère plus néfastes pour l'employé (Brotheridge \& Lee, 2002; Mann, 1999; Schaubroek \& Jones, 2000; Côté \& Morgan, 2002; Brotheridge \& Grandey, 2002; Grandey, 2003). Toutefois, la stratégie de profondeur n'exerce aucun effet ou sinon un effet bénéfique sur ces mêmes conséquences (Grandey, 2003; Brotheridge \& Grandey, 2002; Brotheridge \& Lee, 2003).

Compte tenu de ces effets importants sur les individus, conséquences qui se répercutent par ailleurs sur les organisations de façon plus globale (coûts liés à l'absentéisme et au roulement du personnel, diminution de la qualité de la prestation de service des employés, etc.), il importe de déterminer la nature des antécédents des stratégies de travail émotionnel. Une piste de recherche intéressante émerge en ce sens depuis quelques années. En effet, certains auteurs conceptualisent le travail émotionnel comme un acte motivé (Rubin et al., 2005). La motivation à faire le travail émotionnel est aussi appelé endossement du travail émotionnel (Gosserand \& Diefendorff, 2005). Selon ces derniers auteurs, les employés fournissent des efforts pour afficher et supprimer leurs émotions afin de respecter les exigences de leur emploi dans la mesure où ils endossent le travail émotionnel. En d'autres termes, ils voient le travail émotionnel comme un objectif de travail, et plus cet objectif devient LEUR propre objectif, plus ils adoptent des stratégies de régulation émotionnelle (Gosserand \& Diefendorff, 2005). En lien avec cette notion d'endossement, Kim, Deci et Zuckerman (2002) ont développé une mesure de la motivation autodéterminée à supprimer les émotions négatives dans la vie quotidienne. Cette étude n'a toutefois pas relié la motivation à des stratégies de régulation émotionnelle précise.

Figure 1.

Modèle du processus de régulation émotionnelle de Gross (1998a)

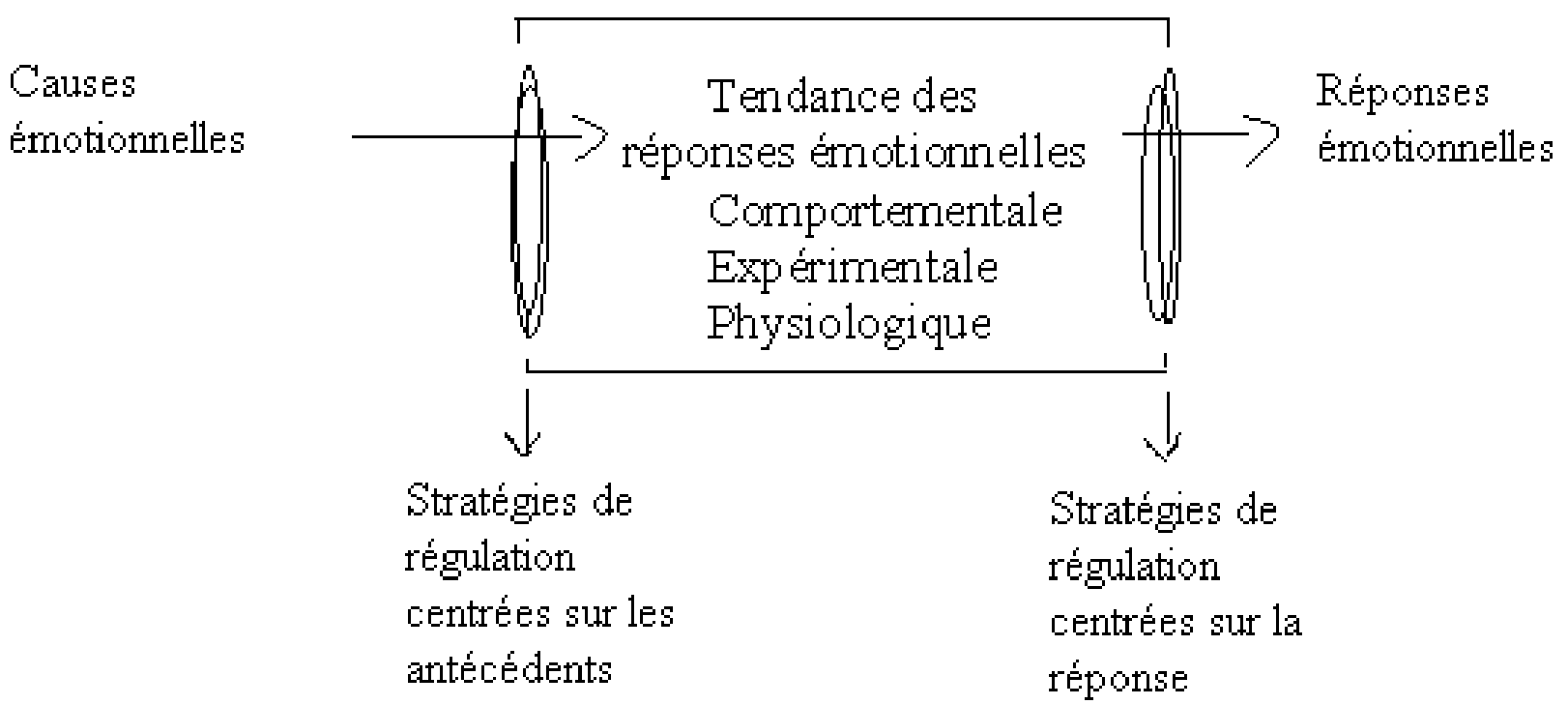

La motivation étant un précurseur important des comportements adoptés par les individus (Vallerand, 1997). Ainsi, nous proposons que la motivation autodéterminée des employés à réguler leurs émotions au travail puisse être un antécédent important aux choix des stratégies de régulation émotionnelle. Les sections suivantes visent à définir le cadre conceptuel de notre mesure, soit la théorie de l'autodétermination. 


\subsection{Théorie de l'autodétermination}

La théorie de l'autodétermination s'intéresse aux différentes formes de motivation. Deci et Ryan (1985) postulent en effet l'existence de six formes de motivation, lesquelles peuvent être positionnées sur un continuum allant de l'amotivation à la motivation intrinsèque. Les formes intermédiaires constituent diverses formes de motivation extrinsèque. Ces différentes formes de motivation ont été évaluées et confirmées dans des études portant sur la motivation générale au travail (voir Gagné et Deci, 2005 pour une recension; Blais et al. 2005), mais aussi, tel que mentionné ci-dessus, dans une étude portant sur la motivation à supprimer ses émotions négatives dans le quotidien (Kim et al., 2002).

Deci et Ryan $(1985 ; 2000)$ distinguent différentes formes de motivation. En effet, le degré de motivation le plus autodéterminé correspond à la motivation intrinsèque. À ce niveau, l'employé agit pour l'intérêt et le plaisir que procurent les activités, d'une façon autonome et qui reflète ses propres choix et décisions. La motivation extrinsèque comporte quant à elle quatre types de motivation: l'externe, l'introjectée, l'identifiée et l'intégrée. Ces motivations sont extrinsèques puisque la personne adopte des comportements pour des raisons instrumentales, telles que satisfaire des attentes ou des objectifs d'une tierce personne, tels qu'un superviseur ou un client. Tel que mentionné ci-dessus, les règles d'affichage prescrites par l'entreprise constituent de telles attentes, lesquelles peuvent être plus ou moins endossée (Gosserand \& Diefendorff, 2005). Dans la terminologie de la théorie de l'autodétermination, les règles d'affichage peuvent être plus ou moins internalisées au soi de l'employé, tel que nous le décrivons cidessous.

Figure 2

Formes de motivation selon la Théorie de l'autodétermination de Deci et Ryan, 1985

\begin{tabular}{|c|c|c|c|}
\hline & Niveau de Motivation & Formes de motivation & Locus \\
\hline \multirow[t]{3}{*}{ Non Autodéterminée } & Amotivation & Absence de motivation & \multirow[b]{2}{*}{ Contrôlante } \\
\hline & \multirow{2}{*}{ Motivation Extrinsèque } & $\begin{array}{c}\text { Externe } \\
\text { Introjectée }\end{array}$ & \\
\hline & & $\begin{array}{l}\text { Identifiée } \\
\text { Intégrée }\end{array}$ & \multirow[b]{2}{*}{ Autonome } \\
\hline Autodéterminée & Motivation Intrinsèque & & \\
\hline
\end{tabular}

Lorsqu'une personne a une motivation externe (Deci \& Ryan, 1985; 2000), ses comportements sont produits afin d'éviter une punition ou pour obtenir une récompense. Dans un contexte de travail émotionnel, les employés motivés de façon externe respecterons les règles d'affichage pour avoir de bonnes évaluations de performance par le superviseur ou encore pour éviter que les clients ne fassent une plainte à leur égard. La motivation externe n'est aucunement internalisée dans le soi de la personne, contrairement aux autres formes de motivation extrinsèques. L'internalisation consiste en une intégration des exigences, attentes ou objectifs définis par d'autres - les règles d'affichage émotionnel dans le contexte de la présente étude - dans le soi de la personne. Le premier niveau d'internalisation est l'introjection. La motivation introjectée signifie que l'employé agit afin d'éviter les sentiments de honte ou d'anxiété qui pourraient survenir s'il ne le faisait pas. Dans ce type de motivation, l'employé s'approprie partiellement le comportement, sans toutefois l'assumer complètement. Les deux motivations expliquées dans ce paragraphe sont des motivations extrinsèques, mais sont dites «contrôlante » puisque la personne n'internalise pas (externe) ou peu (introjection) le comportement à adopter. Le locus de contrôle de la personne est donc externe. 
Les deux autres formes de motivation extrinsèque sont des motivations dites «autonomes ». En effet, bien que la finalité du comportement demeure instrumentale, la personne internalise davantage celui-ci. En d'autres termes, les objectifs fixés par autrui deviennent endossés par la personne. La motivation identifiée survient lorsque la personne endosse le comportement, celui-ci cadrant bien avec certains autres de ses objectifs personnels. Dans le contexte du travail émotionnel, une personne ayant une motivation identifiée régulerait ses émotions parce que cela correspond à ses objectifs personnels au travail. La dernière forme de motivation extrinsèque est la motivation intégrée. La conduite adoptée par l'entremise de cette forme de motivation est cohérente avec les autres valeurs fondamentales de l'individu. Cette forme de motivation est conceptuellement distincte de la motivation intrinsèque. En effet, le comportement à adopter ne procure pas nécessairement du plaisir en tant que tel. Le comportement est adopté pour satisfaire les demandes externes (Deci \& Ryan, 1985; 2000). Finalement, la théorie de l'autodétermination postule une dernière forme de "motivation », soit l'amotivation. Celleci reflète une absence complète de motivation. L'individu agit sans savoir pourquoi et agit machinalement (Deci \& Ryan, 1985; 2000).

Pourquoi prendre la théorie de l'autodétermination pour opérationnaliser la motivation au travail émotionnel? Premièrement, la motivation autodéterminée mène à des conséquences communes au travail émotionnel. En effet, la motivation autodéterminée (intrinsèque ou les formes autonomes de la motivation extrinsèque) mène à des bienfaits (Deci \& Ryan, 1991; Ryan, Deci, \& Grolnick, 1995, Ryan \& Deci, 2000). Deuxièmement, une étude menée par Kim et ses collaborateurs (2002) ont déjà vérifié empiriquement que ce cadre théorique était pertinent pour l'étude de la régulation émotionnelle. Ces auteurs ont également conclut que la motivation autodéterminée à réguler les émotions négatives mène à une meilleure santé psychologique. Troisièmement, ce cadre a également été testé avec succès dans le contexte du travail émotionnel (Cossette, et al., 2006). Cette dernière recherche démontre que la motivation autodéterminée à supprimer les émotions négatives réduit la probabilité d'apparition de l'épuisement émotionnel. Toutefois, cette recherche ne tient compte que d'une seule règle d'affichage. Afin de tenir compte à la fois de la nécessité de supprimer les émotions négatives et d'exprimer des émotions positives dans le cadre des emplois de service à la clientèle, notre recherche propose d'établir une mesure parallèle de la motivation autodéterminée. Plus spécifiquement, l'objectif est d'arriver à mesurer à partir des mêmes énoncés les différentes formes de motivation à exprimer des émotions positives et les différentes formes de motivation à supprimer les émotions négatives.

Cette mesure parallèle contribuerait substantiellement à la littérature sur le travail émotionnel. En effet, à notre connaissance, seule l'étude de Diefendorff et al. (2005) porte sur l'endossement des règles d'affichage par les employés. La mesure utilisée dans cette étude (5 items) comprend toutefois des limites sur le plan conceptuel. En effet, la mesure tient compte simultanément des deux règles d'affichage généralement prônées par les organisations offrant du service à la clientèle. Il est possible que les personnes endossent différemment les règles d'affichage. La mesure de Gosserand et Diefendorff (2005) ne tient pas compte non plus des raisons de cet endossement, à savoir est-ce que la personne endosse les règles d'affichage parce qu'elle en a l'obligation (motivation de type contrôlante) ou au contraire, elle endosse ces règles parce que celles-ci cadrent dans son concept de soi (motivation autonome).

\section{Hypothèses}

Ainsi, un questionnaire sur la motivation à réguler ses émotions au travail a été développé pour la présente étude. Les deux règles d'affichage généralement prescrites en service à la clientèle, soit l'expression des émotions positives et la suppression des émotions négatives, sont présentes dans l'échelle. 
La première question de recherche consiste à déterminer si la théorie de l'autodétermination peut s'appliquer à la notion de travail émotionnel. En d'autres termes, est-ce que les six différentes types de motivation (non autodéterminées et autodéterminées) à réguler ses émotions au travail peuvent être soutenus au niveau de la structure factorielle, et ce, au niveau des deux règles d'affichage émotionnel. Tel que nous l'avons mentionné, l'objectif est d'arriver à créer une échelle de mesure comprenant des énoncées qui évaluent la motivation à afficher des émotions positive d'une part, et la motivation à supprimer des émotions négatives d'autres part.

La deuxième question de recherche, tributaire de la première, concerne la prédiction de l'usage des stratégies de régulation émotionnelle (stratégie de profondeur et de surface) à partir des diverses formes de motivation. En bref, selon la théorie, une personne ayant une motivation davantage autodéterminée ressent moins de contradiction entre ce qu'elle est, ce qu'elle vit et ce qu'elle doit faire dans le cadre de son emploi. Un employé utilisant la stratégie de régulation de profondeur est plus authentique (Brotheridge \& Lee, 2002) car il s'efforce de ressentir les émotions prescrites. Par ailleurs, il est possible que les personnes plus autodéterminées soient portées à faire l'usage de cette stratégie de régulation et qu'elles soient moins enclines à adopter la régulation de surface, stratégie associée à un haut niveau de dissonance émotionnelle (Grandey, 2000; 2003) ou d'inauthenticité. Par opposition, les personnes moins autodéterminées devraient adopter davantage la stratégie de surface et moins la stratégie de profondeur. Finalement, les personnes amotivées ne devraient pas avoir recours aux stratégies de régulation émotion de profondeur ou de surface, ces personnes étant dépourvues d'intention quant à la régulation émotionnelle. Plus formellement, les hypothèses de recherche sont les suivantes, lesquelles sont tributaires de la confirmation des formes de motivation postulées par la théorie de l'autodétermination.

Hypothèse 1 : La motivation intrinsèque sera associée a) positivement à la stratégie de profondeur et b) négativement à la stratégie de surface.

Hypothèse 2: La motivation contrôlante (régulation externe et introjectée) sera a) négativement associée à la stratégie de profondeur et b) positivement associée à la stratégie de surface.

Hypothèse 3: La motivation autonome (régulation identifiée et intégrée) sera positivement liée a) à la stratégie de profondeur et b) négativement à la stratégie de surface.

Hypothèse 4: L'amotivation sera négativement associée à l'usage des stratégies a) de profondeur et b) de surface.

\section{Méthodologie}

\section{1 Échantillon}

Les participants sont des étudiants universitaires. Des 223 participants, 72,32\% sont des femmes, $24,55 \%$ sont des hommes et 3,13\% n'ont pas répondu. La moyenne d'âge est de 26,06 ans (ET=7,55 ans). En moyenne, les participants travaillaient depuis 4,05 ans dans l'entreprise sur laquelle ils ont basé leurs réponses ( $E T=4,44$ ans). Pour cette étude, des étudiants ont été sollicités pour participer volontairement. Les répondants devaient travailler dans le domaine du service à la clientèle.

\subsection{Procédure, matériel et validation du matériel}

L'échelle de mesure de la motivation à réguler les émotions au travail a été développée en se basant sur les énoncés de Forest, Gagné, Vansteenkiste, et al. (2010) sur la motivation générale au travail. 
L'échelle de Forest et al. (2010) permet de mesurer quel est le type de motivation des employés, décrit par la théorie de l'autodétermination. Une recension des échelles motivationnelles déjà existantes dans différents contextes tels que l'éducation et le sport, a également été faite afin de bien saisir la formulation reconnue des énoncés de chacun des types de motivation.

Un questionnaire de 67 énoncés sur les types de motivation de la théorie de l'autodétermination a été développé. Chaque énoncé correspond à une raison pouvant expliquer pourquoi les gens régulent leurs émotions au travail. Ces raisons reflètent les différentes formes de motivation expliquée ci-dessus. Voici un exemple d'énoncé pour chacun des niveaux de motivation : «Parce que j'aime vraiment ce travail » (intrinsèque), «Car je suis fait pour ce travail » (intégrée), «Parce qu'il est important pour moi de le faire lorsque j'interagis avec les clients » (identifiée), «Car je considère cela comme étant mon devoir envers mon entreprise » (introjectée), «Car mes collègues réagiraient mal si je n'y parvenais pas » (externe) et «Je le fais peu, car j'ai vraiment l'impression de perdre mon temps à faire ce travail » (amotivation). Les participants devaient répondre à chaque énoncé sur une échelle de Likert à 7 points, « 1 » correspondant à «pas du tout pour cette raison » et « 7 » à « exactement pour cette raison ». Les participants pouvaient cocher la case «non applicable » s’ils étaient inconfortables avec les énoncés.

Afin de mesurer les stratégies de régulation émotionnelle, les neuf énoncés de Brotheridge et Lee (2002) sur l'usage des stratégies de régulation (régulation de surface et régulation de profondeur) ont été utilisés. Voici un exemple d'énoncé pour chaque type de stratégie : «Je démontre des émotions que je ne ressens pas avec mes clients » (stratégie de surface) et «J'essaie de vivre concrètement les émotions que je dois absolument démontrer avec mes clients » (stratégie de profondeur).

\section{Résultats}

\subsection{Théorie de l'autodétermination appliquée à la motivation au travail émotionnel}

Le premier objectif de la recherche est de confirmer la présence des types de motivation postulés par la théorie de l'autodétermination à partir d'une série d'énoncés. Afin de conserver des énoncés qui s'avèrent compréhensibles aux yeux des participants, nous avons éliminé des analyses subséquentes les énoncés ayant plus de 5\% de réponses manquantes (aucune réponse donnée ou lorsque la réponse du participant était «non applicable ». Chaque énoncé restant a fait l'objet d'une analyse descriptive afin d'en vérifier la distribution. Aucune anomalie n'a été observée en termes de moyenne, d'écart-type, d'étendue, d'aplatissement et de symétrie.

Afin de répondre à la première question de recherche (est-ce que la théorie de l'autodétermination peut s'appliquer au travail émotionnel), des analyses factorielles confirmatoires ont été réalisées sur les énoncés. Les analyses initiales n'ont pas permis d'obtenir un ajustement acceptable, tant pour la motivation à exprimer des émotions positives (MEEP) que pour la motivation à supprimer les émotions négatives (MSEN). Une série d'analyses factorielles confirmatoires nous a permis de constater que les items mesurant la motivation intrinsèque étaient ceux qui posaient des problèmes dans l'ajustement des modèles. Ces énoncés ont donc été retirés des analyses. Outre cette justification statistique, force est de constater que la motivation intrinsèque est un concept inapplicable à la notion de travail émotionnel. Par définition, le travail émotionnel constitue une demande organisationnelle quant à l'affichage ou la suppression d'émotions. Le locus de la tâche est donc externe à la personne, bien qu'elle puisse être internalisée à des degrés divers.

À partir d'autres analyses factorielles confirmatoires, des énoncés ont été retirés à cause de leur saturation factorielle trop faible ou parce qu'ils étaient trop fortement corrélés à d'autres types de motivation que celle postulée. Concernant l'analyse finale des énoncés mesurant la MEEP, l'ajustement 
du modèle est adéquat, $\chi_{(160)}^{2}=295,25, p<, 001, \mathrm{Cmin} / \mathrm{dl}=1,85, \mathrm{GFI}=, 88, \mathrm{CFI}=, 93, \mathrm{RMSEA}=, 06$. En ce qui a trait à la MSEN, l'ajustement est également adéquat, $\chi_{(160)}^{2} 316,25, p<, 001, \mathrm{Cmin} / \mathrm{dl}=1,98$, GFI $=, 88, \mathrm{CFI}=, 91$ et RMSEA $=0,07$. Rappelons que seuls les énoncés qui étaient statistiquement valides à la fois pour la MEEP que pour la MSEN ont été maintenus dans ces échelles finales. Pour chacun des types de motivation, une analyse de consistance interne a été réalisée. Chacun des types de motivation obtient des résultats satisfaisants (voir alpha de Cronbach aux tableaux I et II).

En somme, les résultats aux diverses analyses statistiques soutiennent la fiabilité et la validité des échelles. En conséquence, 22 énoncés ont été retenus afin de former l'échelle de mesure parallèle de la motivation à réguler les émotions au travail. Afin de poursuivre l'exploration de la motivation au travail émotionnel, nous avons constitué de nouvelles variables reflétant les différents construits motivationnels.

Ayant des motivations de nature bien différentes (absence de motivation, motivations contrôlante et autonome), les questions suivantes se posent : quel type de motivation est le plus important chez les employés participant à la recherche et est-ce que les types de motivation sont modulés selon le sexe des employés et selon leur niveau de scolarité (note : seules les personnes ayant un diplôme collégial ou universitaire sont inclus dans les analyses subséquentes, les personnes détenant un niveau primaire ou secondaire n'étant pas assez nombreux $[n=7]$ ). Pour répondre à cette question, des analyses de variance à un facteur répété (amotivation, motivation contrôlante et motivation autonome) et à deux facteurs non répétés (sexe et scolarité) indiquent que la motivation autonome $\left(M_{\text {meep }} 5,12=; S D_{\text {meep }}=1,16 ; M_{\text {msen }}=\right.$ 4,$\left.40 ; S D_{\text {msen }}=1,28\right)$ est la plus élevée des motivations, suivi de la motivation contrôlante $\left(M_{\text {meep }}=3,62\right.$; $\left.S D_{\text {meep }}=1,81 ; M_{\text {msen }}=3,85 ; S D_{\text {men }}=1,21\right)$ et de l'amotivation $\left(M_{\text {meep }}=1,61 ; S D_{\text {meep }}=0,98 ; M_{\text {msen }}=1,77\right.$; $\left.S D_{\text {msen }}=1,01\right)$, et ce, tant pour la $\operatorname{MEEP}(F(1,188)=208,3, p<, 001)$ que pour la $\operatorname{MSEN}(F(1,184)=$ $107,0, p<, 001)$. Seule l'interaction entre le type de MSEN et le niveau de scolarité $(F(4,370)=2,6, p=$ ,04) s'avère significative. En décomposant cette interaction, les résultats indiquent que la scolarité n'influence pas le niveau de MSEN-amotivation $(F(2,190)=0,5, p=, 62)$ ou la MSEN contrôlante $(F(2,199)=2,0, p=, 14)$. Par contre, la MSEN autonome varie légèrement selon la scolarité. En effet, les personnes ayant un baccalauréat $(M=4,70 ; S D=1,29)$ obtiennent une MSEN autonome légèrement supérieure à celle des personnes détenant un diplôme collégial $(M=4,18 ; S D=1,25)(p<, 05)$. La MSEN autonome des personnes ayant une maîtrise ou un doctorat $(M=4,27 ; S D=1,34)$ ne diffère toutefois pas de celle des personnes ayant un baccalauréat ou un diplôme collégial $(p s>, 05)$.

En somme, dans l'ensemble, les participants ont davantage une motivation autodéterminée au travail émotionnel. En d'autres termes, ils endossent la nécessité de réguler leurs émotions dans le cadre de leur travail, mais comprennent aussi que ce travail émotionnel doit être fait pour satisfaire les clients et les exigences organisationnelles.

\subsection{Stratégies de travail émotionnel et motivation}

Le dernier objectif de la recherche visait à déterminer si les types de motivation peuvent prédire le recours aux stratégies de régulation émotionnelle. Afin de procéder à l'examen des hypothèses spécifiques reliant les formes de motivation aux stratégies de régulation, des analyses de régression multiples ont été effectuées ${ }^{1}$. Chaque régression a pour variable dépendante une stratégie de régulation émotionnelle. Les corrélations entre les variables sont présentées au Tableau I pour la MEEP et au Tableau II pour la MSEN.

\footnotetext{
${ }^{1}$ L'hypothèse reliant la motivation intrinsèque aux stratégies de régulation n'a pu être testée étant donnée l'exclusion de cette dimension.
} 


\section{Tableau I}

Motivation à exprimer des émotions positives : corrélations et coefficients de consistance interne ( $\alpha)$

\begin{tabular}{lccccccc}
\hline & $M$ & $E T$ & 1. & 2. & 3. & 4. & 5. \\
1. Amotivation & 1,63 & 1,00 & $\mathbf{, 8 2}$ & & & & \\
2. Externe & 4,00 & 1,39 &, $18 * *$ & $\mathbf{8 1}$ & & & \\
3. Introjectée & 3,16 & 1,56 &, 03 &, $35 * *$ & $\mathbf{, 8 8}$ & & \\
4. Identifiée & 5,41 & 1,15 &,$- 26 * *$ &, $20 * *$ &, $28 * *$ &, 76 & \\
5. Intégrée & 4,80 & 1,37 &,$- 21 * *$ &, 04 &, $39 * *$ &, $67 * *$ & $\mathbf{6 9}$ \\
\hline
\end{tabular}

** $p<, 01$ (bilatéral)

Tableau II

Motivation à supprimer des émotions négatives : corrélations et coefficients de consistance interne ( $\alpha)$

\begin{tabular}{lccccccc}
\hline & $M$ & $E T$ & 1. & 2. & 3. & 4. & 5. \\
1. Amotivation & 1,81 & 1,06 & $\mathbf{, 8 2}$ & & & & \\
2. Externe & 4,14 & 1,44 &, 04 & $\mathbf{8 0}$ & & & \\
3. Introjectée & 3,47 & 1,59 &, 00 &, $37 * *$ & $\mathbf{, 8 6}$ & & \\
4. Identifiée & 4,80 & 1,36 &,$- 32 * *$ &, $25 * *$ &, $30 * *$ & $\mathbf{6 5}$ & \\
5. Intégrée & 3,99 & 1,53 &,- 13 &, 10 &, $45 * *$ &, $64 * *$ &, 74 \\
\hline
\end{tabular}

$* * \mathrm{p}<, 01$ (bilatéral)

Les variables sociodémographiques ont été contrôlées afin de dégager l'effet des différentes motivations. L'âge est systématiquement et inversement relié aux deux stratégies de régulation émotionnelle. En outre, les résultats des régressions démontrent que l'Hypothèse 2 est partiellement confirmée (Tableau III). En effet, la stratégie de surface est uniquement liée à la motivation contrôlante concernant les deux règles d'affichage.

Le Tableau IV présente les résultats de la stratégie de profondeur. La MEEP contrôlante est positivement liée à la stratégie de profondeur tout comme pour la MSEN contrôlante, ce qui invalide partiellement l'Hypothèse 2. La motivation autonome est significativement liée à la stratégie de profondeur pour les deux règles d'affichage, confirmant l'Hypothèse $3 \mathrm{a}$.

Tableau III

Analyse de régression: Stratégie de surface $(\beta)$

\begin{tabular}{lcccc}
\hline \multirow{2}{*}{$\begin{array}{l}\text { Variables } \\
\text { indépendantes }\end{array}$} & \multicolumn{4}{c}{ Stratégie de surface } \\
\cline { 2 - 5 } & \multicolumn{2}{c}{ Exprimer ses émotions } & \multicolumn{2}{c}{ Supprimer ses émotions } \\
nositives & Égatives \\
Âge 1 &,$- 190 *$ & Étape 1 & Étape 2 \\
Sexe &,$- 218 *$ &,- 002 &,$- 239 *$ &,$- 215 *$ \\
Scolarité &,- 038 &, 043 &, 040 &, 001 \\
Durée emploi &, 022 &, 171 &, 163 &, 039 \\
Durée du poste &, 144 &,- 132 &,- 117 &, 154 \\
Amotivation &,- 113 &, 098 & &,- 126 \\
Contrôlante & &, $277 * * *$ & &, $228 * *$ \\
Autonome & &,- 109 & &, 097 \\
\hline
\end{tabular}

$* \mathrm{p}<, 05 \quad * * \mathrm{p}<, 01 \quad * * * \mathrm{p}<, 001$ 
Tableau IV

Analyse de régression : Stratégie de profondeur $(\beta)$

\begin{tabular}{|c|c|c|c|c|}
\hline \multirow{3}{*}{$\begin{array}{l}\text { Variables } \\
\text { indépendantes }\end{array}$} & \multicolumn{4}{|c|}{ Stratégie de profondeur } \\
\hline & \multicolumn{2}{|c|}{$\begin{array}{l}\text { Exprimer ses émotions } \\
\text { positives }\end{array}$} & \multicolumn{2}{|c|}{$\begin{array}{c}\text { Supprimer ses émotions } \\
\text { négatives }\end{array}$} \\
\hline & Étape 1 & Étape 2 & Étape 1 & Étape 2 \\
\hline Âge &,$- 263 * *$ &,$- 267 * *$ &,$- 261 * *$ &,$- 243 * *$ \\
\hline Sexe & ,007 &, 083 & 019 & ,067 \\
\hline Scolarité &,- 020 &,- 014 &,- 015 &,- 011 \\
\hline Durée emploi &,- 033 &,- 021 &,- 010 &,- 039 \\
\hline Durée du poste &, 070 &, 071 & ,002 & 000 \\
\hline Amotivation & &, 085 & & 035 \\
\hline Contrôlante & &, $338 * * *$ & &, $218 * *$ \\
\hline Autonome & &, $155 *$ & &, $174 *$ \\
\hline
\end{tabular}

\section{Discussion}

Initialement, trois objectifs ont été formulés pour la présente étude. Le premier était de déterminé si la théorie de l'autodétermination peut être appliquée au concept de la régulation émotionnel au travail (travail émotionnel). Le deuxième objectif poursuivi était de produire une échelle de mesure qui évaluerait la motivation à se conformer aux deux règles d'affichage à l'aide des mêmes énoncées. En d'autres termes, il s'agissait d'élaborer une mesure parallèle. Enfin, le troisième objectif visait à relier les diverses formes de motivations aux stratégies de régulation émotionnelle.

Les résultats révèlent que la théorie de l'autodétermination s'applique au travail émotionnel. En effet, les analyses factorielles confirmatoires soutiennent globalement la structure dimensionnelle. De plus, les corrélations entre les niveaux motivationnels rapprochés sont élevées alors que les corrélations entre niveaux plus éloignés sont non significatives ou inversement reliées, soutenant ainsi globalement le continuum de l'autodétermination (Gagné \& Deci, 2005). Ces résultats sont valables à la fois pour la motivation à exprimer les émotions positives et pour la motivation à supprimer les émotions négatives. Par contre, deux corrélations s'avèrent problématiques, soit celles entre les motivations «intégrée » et « introjectée », tant pour la motivation à exprimer des émotions positives que la motivation à supprimer les émotions négatives. Il était attendu que ces corrélations soient inversement corrélées ou non significatives or, elles sont significatives et positivement corrélées. Ces résultats peuvent s'expliquer par la difficulté à différencier ces deux types de motivation par les répondants. En effet, la motivation introjectée sous-tend une certaine affectivité chez l'employé. Celle-ci a pu être confondue par les répondants avec les émotions impliquées dans les stratégies de régulation émotionnelle. Les études futures devraient s'attarder à cette relation entre les deux dimensions.

Le recourt aux deux stratégies de régulation émotionnelle peut être prédit par le type de motivation et l'âge de l'employé. Initialement, il était attendu que la motivation intrinsèque soit positivement associée à la stratégie de profondeur et négativement avec la régulation de surface (H1). Or, la motivation intrinsèque a été retirée des analyses puisqu'elle nuisait aux structures factorielles. Une explication probable à ce résultat est que le travail émotionnel est avant toute chose accompli afin de satisfaire une demande externe. Or, la motivation intrinsèque réfère à l'accomplissement d'une tâche pour l'intérêt et le plaisir qu'elle procure. Elle implique donc une absence de demande externe à la personne.

Bien que l'Hypothèse 1 n'ait pu être soutenue, les résultats ont permis de confirmer certaines autres hypothèses. En effet, il était prédit que la motivation contrôlante soit positivement associée à la 
suppression et à la simulation émotionnelle, et négativement liée à la stratégie de profondeur (H2). Seul le lien positif avec la stratégie de surface a été confirmé. Le lien avec la stratégie de profondeur fut également positif, contrairement à l'hypothèse. Ce résultat inattendu entre la motivation contrôlante et la stratégie de profondeur peut s'expliquer par la formulation des énoncés de Brotheridge et Lee (2002). En effet, la formulation insiste sur l'obligation de réguler les émotions, ce qui porte à croire qu'il y ait d'emblée une cohérence entre cette stratégie de régulation et la motivation contrôlante.

Il était également attendu que la motivation autonome (identifiée et intégrée) soit positivement associée à la stratégie de profondeur et négativement liée à la stratégie de surface (H3). Encore une fois, cette hypothèse n'est que partiellement confirmée; la motivation autonome n'est significativement liée qu'avec la stratégie de profondeur. Toutefois, l'absence d'effet significatif avec la stratégie de surface peut s'expliquer avec les résultats d'une autre étude (Cossette \& Hess, 2010). Selon cette étude, certains employés ayant une motivation autodéterminée font l'usage de la stratégie de surface ainsi que d'autres stratégies, selon la situation. Il est donc possible que la régulation de surface serve à titre de stratégie ponctuelle, alors que le recours à la régulation de profondeur soit plus systématique chez ces personnes.

Concernant l'amotivation, il était attendu qu'elle soit négativement associée aux stratégies de régulation émotionnelle $(\mathrm{H} 4)$. Cette hypothèse n'est pas soutenue. Le faible niveau d'amotivation chez les participants peut expliquer l'absence de relation avec les stratégies de régulation. L'absence de relation significative peut également suggèrer que certaines personnes amotivées régulent leurs émotions alors que d'autres personnes amotivées ne le font pas. Les recherches futures devraient s'attarder davantage aux personnes amotivées afin de mieux comprendre leurs usages des stratégies de régulation.

Enfin, l'âge a été systématiquement et négativement associé à l'usage des stratégies de régulation. Ce dernier résultat peut s'expliquer d'au moins deux façons. Premièrement, les personnes plus âgées peuvent ressentir plus spontanément les émotions prescrites par l'organisation et ainsi avoir moins besoin de réguler leurs émotions. Deuxièmement, il est possible que les personnes plus âgées aient l'habitude de réguler les émotions. En conséquence, le comportement est devenu plus évident à accomplir et nécessiterait moins d'efforts (Zapf, 2002). Dans cette seconde perspective, l'individu aurait internalisé les deux règles d'affichage et effectuerait le travail émotionnel automatiquement.

\subsection{Limites et perspectives futures}

Comme toute étude, certains biais peuvent limiter la portée des résultats. Premièrement, l'intérêt que les participants vouent à leur emploi peut avoir eu une influence sur leur niveau de motivation. En effet, l'échantillon étant composé d'étudiants universitaires, il est possible qu'ils n'accordent pas un intérêt aussi marqué envers leur travail qu'une personne travaillant à temps plein. Il serait intéressant d'examiner l'interrelation entre la motivation générale au travail et la motivation à réguler les émotions afin d'obtenir un portrait plus complet du profil motivationnel de la personne et de l'impact sur les stratégies de régulation émotionnelle.

Deuxièmement et lié à la première limite, il serait pertinent d'utiliser la mesure auprès d'employés travaillant à temps plein et appartenant à un groupe d'emplois plus homogènes afin d'assurer la généralisation des résultats. Ceux-ci ne tiennent pas compte du type d'emploi. De ce fait, le type de rémunération et la nature des interactions (face à face ou par téléphone) peuvent avoir influencé les résultats. Cette limite ouvre la porte à une investigation en ce sens, à savoir l'impact des pratiques de gestion des ressources humaines de façon générale ou du climat de travail de façon plus particulière sur la motivation au travail émotionnel. 
Troisièmement, il serait intéressant de lier la motivation à réguler les émotions à d'autres variables telles que des antécédents (personnalité, climat de travail, etc.) ou des conséquences (santé psychologique, attitudes au travail). Cette piste de recherche permettrait de vérifier le réseau nomologique du travail émotionnel.

Finalement, concernant l'échelle de mesure, puisqu'un seul questionnaire a servi à mesurer les variables indépendantes et la variable dépendante, il peut avoir un biais de variance commune. De ce fait, l'ampleur des relations révélées par les analyses corrélationnelles peut être gonflée.

\section{Conclusion}

Ces limites ne doivent pas porter ombrage aux contributions de l'étude. Celle-ci a permis d'opérationnaliser la motivation autodéterminée à réguler les émotions au travail, offrant un nouvel appui à la théorie de l'autodétermination. Nous sommes donc parvenus à élaborer et évaluer une échelle de mesure parallèle de la motivation à réguler les émotions, incluant les deux règles d'affichage généralement prescrites par les entreprises dans le domaine du service à la clientèle - exprimer des émotions positives et supprimer des émotions négatives. En outre, la motivation permet d'expliquer l'utilisation des diverses stratégies de régulation émotionnel au travail. La notion de motivation à réguler les émotions ouvre donc la voie à des recherches prometteuses.

\section{Références}

Abraham, R. (1998). Emotional dissonance in organizations: antecedents, consequences, and moderators. Genetic, Social, and General Psychology Monographs, 124, 229-246.

Abraham, R. (1999). The impact of emotional dissonance on organizational commitment and intention to turnover. Journal of Psychology: Interdisciplinary and Applied, 133, 441-455.

Ashforth, B. E., \& Humphrey, R. H. (1993). Emotional labor in service roles: the influence of identity. Academy of Management Review, 18, 88-115.

Blais, M.R., Brière, N.M., Lachance, L., Riddle, A.S. \& Vallerand, R.J. (1993). L'inventaire des motivations au travail de Blais, Revue québécoise de psychologie, 14 (3), 185-215.

Brotheridge, C. M., \& Grandey, A. A. (2002). Emotional labor and burnout: comparing two perspectives of "people work". Journal of Vocational Behavior, 60, 17-39.

Brotheridge, C.M., \& Lee, R.T. (1998). Development and validation of the emotional labor scale. Paper presented at the First Conference on Emotions in Organizational Life, San Diego, CA.

Brotheridge, C. M., \& Lee, R. T. (2002). Testing a conservation of resources model of the dynamics of emotional labor. Journal of Occupational Health Psychology, 7, 57-67.

Brotheridge, C. M., \& Lee, R. T. (2003). Development and validation of the emotional labour scale. Journal of Occupational and Organizational Psychology, 76, 365.

Chu, K.H-L., \& Murmann, S.K. (2006). Development and validation of the hospitality emotional labor scale. Tourism Management 27 (6), 1181-1191.

Cossette, M., Blais, M., \& Hess, U. (2006). Motivation au travail émotionnel, styles de service à la clientèle et épuisement professionnel. Papier présenté au $14^{\mathrm{e}}$ Congrès de psychologie du travail et des organisations (AIPTLF)

Cossette, M., \& Hess, U. (2010) How is Emotional Labor Performed by Customer Service Employees? A Matter of Style. Academy of Management annual meeting. Montreal, Canada, 6 au 10 août 2010.

Cossette, M. (2008) Styles de régulation émotionnelle des employés de service : antécédents organisationnels, motivation et conséquences sur la santé psychologique. Thèse de doctorat non publiée, Université du Québec à Montréal, Canada.

Côté, S., \& Morgan, L. (2002). A longitudinal analysis of the association between emotion regulation, job satisfaction, and intentions to quit. Journal of Organizational Behavior, 23, 947-962.

Deci, E. L., \& Ryan, R. M. (1985). Intrinsic motivation and self-determination in human behavior. New York: Plenum Press. 
Deci, E. L., \& Ryan, R. M. (1991). A motivational approach to self: Integration in personality. In R. Dienstbier (Ed.), Nebraska symposium on motivation: perspectives on motivation, vol. 38 (pp. 237-288). Lincoln, NE: University Of Nebraska Press.

Deci, E. L., \& Ryan, R. M. (1995). Human autonomy: The basis for true self-esteem. In M. Kernis (Ed.), Efficacy, agency, and self-esteem (pp. 31-49). New York: Plenum Publishing Co.

Deci, E. L., \& Ryan, R. M. (2000). The "what" and "why" of goal pusuits: human needs and self-determination of behavior. Psychological Inquiry, 11, 227-268.

Deci, E. L., \& Ryan, R. M. (1996-2008). Self-Determination Theory (SDT) An approach to human motivation and personnality. University of Rochester, retrouvé le 3 mars 2010, disponible sur http://www.psych.rochester.edu/SDT/index.php

Diefendorff, J. M., Croyle, M. H., \& Gosserand, R. H. (2005). The dimensionality and antecedents of emotional labor strategies. Journal of Vocational Behavior, 66, 339.

Diefendorff, J. M., \& Gosserand, R. H. (2003). Understanding the emotional labor process: a control theory perspective. Journal of Organizational Behavior, 24, 945-959.

Diefendorff, J.M., Richard, E.M., \& Yang, J. (2008). Linking emotion regulation strategies to affective events and negative emotions at work. Journal of Vocational behavior, 73, 498-508

Forest, J., Gagné, M., Vansteenkiste, M., Van den Broeck, A., Crevier-Braud, L., Bergeron, E., Benabou, C., Nuñez, J. L., Martin-Albo, J., Battistelli, Picci, P., Galletta, M., Naudin, M., \& Mans, N. (2010). International validation of the "Revised Motivation at Work Scale": Validation evidence in five different languages (French, English, Italian, Spanish, \& Dutch). Présentation orale dans le cadre du 4e congrès international sur la Théorie de l'autodétermination, à Ghent, en Belgique.

Gagné, M., \& Deci, E. L. (2005). Self-determination theory and work motivation. Journal of Organizational Behavior, 26, 331-362.

Glomb, T. M., \& Tews, M. J. (2004). Emotional labor: a conceptualization and scale development. Journal of Vocational Behavior, 64, 1-23.

Gosserand, R. H., \& Diefendorff, J. M. (2005). Emotional display rules and emotional labor: the moderating role of commitment. Journal of Applied Psychology, 90, 1256-1264.

Grandey, A. A. (2000). Emotional regulation in the workplace: a new way to conceptualize emotional labor. Journal of Occupational Health Psychology, 5, 95-110.

Grandey, A. A. (2003). When "the show must go on": surface acting and deep acting as determinants of emotional exhaustion and peer-rated service delivery. Academy of Management Journal, 46, 86.

Gross, J.J. (1998 a). Antecedent- and response-focused emotion regulation: Divergent consequences for experience, expression, and physiology. Journal of Personality and Social Psychology, 74, 224-237.

Gross, J. J. (1998 b). The emerging field of emotion regulation: an integrative review. Review of General Psychology. Special Issue: New directions in research on emotion, 2, 271-299.

Gross, J. J., \& John, O. P. (2003). Individual differences in two emotion regulation processes: implications for affect, relationships, and well-being. Journal of Personality and Social Psychology, 85, 348-362.

Hess, U. Rapport bourgogne, Les émotions au travail. CIRANO, Retrouvé le 8 août 2010, disponible sur http://www.cirano.qc.ca/pdf/publication/2003RB-04.pdf

Hochschild, A. R. (1983). The managed heart: commercialization of human feeling. Berkeley and Los Angeles: University of California Press.

Kim, H.J. (2008). Hotel service providers' emotional labor; antecedents and effects on burnout. International Journal of Hospitality Management 27 (2), 151-161

Kim, Y., Deci, E. L., \& Zuckerman, M. (2002). The development of the self-regulation of withholding negative emotions questionnaire. Educational and Psychological Measurement, 62, 316-336.

Kruml, S. M., \& Geddes, D. (2000). Exploring the dimensions of emotional labor. Management Communication Quarterly : $M c Q, 14,8-49$.

Mann, S. (1999). Emotion at work: to what extent are we expressing, suppressing, or faking It? European Journal of Work and Organizational Psychology, 8, 347-369.

Maslach, C., \& Jackson, S. E. (1984). Burnout in organizational settings. Applied Social Psychology Annual, 5, 133153.

Morris, J. A., \& Feldman, D. C. (1996a). The dimensions, antecedents, and consequences of emotional labor. Academy of Management Review, 21, 986-1010.

Morris, J. A., \& Feldman, D. C. (1996b). The impact of emotional dissonance on psychological well-being: the importance of role internalization as a mediating variable. Management Research News, 19, 19-28. 
Pugliesi, K. (1999). The consequences of emotional labor: effects on work stress, job satisfaction, and well-being. Motivation and Emotion. Special Issue: Sociological contributions to the understanding of emotion, 23, 125154.

Rafaeli, A. \& Sutton, R.I. (1987). The expression of emotion as part of the work role. Academy management review, 12, 23-37.

Ryan, R. M., Deci, E. L., \& Grolnick, W. S. (1995). Autonomy, relatedness, and the self: Their relation to development and psychopathology. In D. Cicchetti \& D. J. Cohen (Eds.), Developmental psychopathology: Theory and methods (pp. 618-655). New York: Wiley

Schaubrock, J., \& Jones, J.R. (2000). Antecedents of workplace emotional labor dimensions and moderators of their effects on physical symptoms. Journal of Organizational Bahavior, 21, 163-183.

Vallerand, R. J. (1997). Toward a hierarchical model of intrinsic and extrinsic motivation. In M.P. Zanna (Ed.), Advances in experimental social psychology (pp. 271-360). New York: Academic Press.

Van Dijk, P.A., \& Brown, A.K. (2006). Emotional labour and negative job outcomes: An evaluation of the mediating role of emotional dissonance. Journal of Management and Organization, 12, 101-115.

Zapf, D. (2002). Emotion work and psychological well-being. A review of the literature and some conceptual considerations. Human Ressource Management Review, 12, 237-268.

Zapf, D., Vogt, C., Seifert, C., \& Holz, M. (2001). Emotion work and job stressors and their effects on burnout. Psychology and Health, 16, 527-545. 